\title{
Acknowledgement to Reviewers of Non-Coding RNA in 2017
}

\section{Non-Coding RNA Editorial Office}

MDPI AG, St. Alban-Anlage 66, 4052 Basel, Switzerland

Published: date

Peer review is an essential part in the publication process, ensuring that Non-Coding RNA maintains high quality standards for its published papers. In 2017, a total of 24 papers were published in the journal. Thanks to the cooperation of our reviewers, the median time to first decision was 25.50 days and the median time to publication was 62.50 days. The editors would like to express their sincere gratitude to the following reviewers for their time and dedication in 2017:

\begin{tabular}{ll} 
Alvarez-Dominguez, Juan R. & Hamamah, Sameer \\
Aniello, Francesco & Hiroshi, Nakayama \\
Atianand, Maninjay K. & Janga, Madhusudhana \\
Bacolla, Albino & Janitz, Michael \\
Bazzini, Ariel & Jiang, Wei \\
Benistant, Christine & Jin, Hailing \\
Biswas, Santanu & Jordan, King \\
Blanco, Enrique & Kav, Nat N. V. \\
Blin, Kai & Kim, Taewan \\
Bolger, Anthony & Kosaka, Nobuyoshi \\
Borges, Filipe & Kotsikorou, Evangelia \\
Bose, Debojit & Kuchenbauer, Florian \\
Budak, Hikmet & Kumar, Rajesh \\
Chakrabarti, Kausik & Kumar, Rajive \\
Chakrabarti, Kausik & Li, Hongda \\
Chiang, Tai-an & Li, Lin \\
Ciaudo, Constance & Lin, Chin-Yo \\
Cock, J. Mark & Liu, Changning \\
D'Agnano, Igea & Liu, Jun \\
Dibb, Nicholas & Melters, Daniel \\
Dimri, Goberdhan & Melters, Daniel \\
Donato, Michele & Mo, Yin-Yuan \\
Dragomir, Andrei & Moran, Yehu \\
Dweep, Harsh & Moreno-Mateos, Miguel \\
Enguita, Francisco & Mueller, Soeren \\
Fava, Eugenio & Necsulea, Anamaria \\
Ferracin, Manuela & Nelson, Andrew \\
Ferreira, Luciana & Nowicki, Marcin \\
Fiannaca, Antonino & Oler, Andrew \\
García-Escudero, Ramón & Panda, Amaresh \\
González, José M. & Pathania, Rajneesh \\
Gupta, Sachin Kumar & Pichler, Martin \\
& \\
\hline
\end{tabular}


Sanchez-Carbayo, Marta

Schmittgen, Thomas

Singh, Kasmir

Slotkin, Keith

Stathopoulos, Constantinos

Sterne-Weiler, Tim

Sutaria, Dhruvitkumar

Taguchi, Yoshihiro

Tesfaye, Dawit

Uchida, Shizuka

Uchida, Shizuka

Unver, Turgay

Unver, Turgay

Valdmanis, Paul
VanderWeele, David

Vaughan, Ashley

Volders, Pieter-Jan

Volders, Pieter-Jan

Vora, Mehul

Wang, Jiguan

Wang, Lixiao

Wang., Kai

Xiao, Jun

Xuan, Zhenyu

Yakovlev, Igor A.

Yamada, Masashi

Yu, Xiang

(C) 2018 by the authors. Submitted for possible open access publication under the terms and conditions of the Creative Commons Attribution (CC-BY) license (http://creativecommons.org/licenses/by/4.0/). 